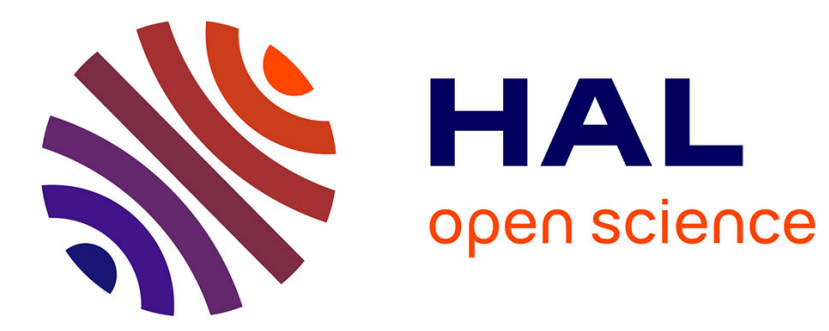

\title{
Comparison of Direct Multiobjective Optimization Methods for the Design of Electric Vehicles
}

\author{
Xavier Roboam, Jérémi Regnier, Bruno Sareni, Yan Fefermann
}

\section{To cite this version:}

Xavier Roboam, Jérémi Regnier, Bruno Sareni, Yan Fefermann. Comparison of Direct Multiobjective Optimization Methods for the Design of Electric Vehicles. EPE Journal - European Power Electronics and Drives, 2006, vol. 16 ( $\mathrm{n}^{\circ} 3$ ), pp. 1-18. hal-01179106

\section{HAL Id: hal-01179106 https://hal.science/hal-01179106}

Submitted on 22 Jul 2015

HAL is a multi-disciplinary open access archive for the deposit and dissemination of scientific research documents, whether they are published or not. The documents may come from teaching and research institutions in France or abroad, or from public or private research centers.
L'archive ouverte pluridisciplinaire HAL, est destinée au dépôt et à la diffusion de documents scientifiques de niveau recherche, publiés ou non, émanant des établissements d'enseignement et de recherche français ou étrangers, des laboratoires publics ou privés. 


\title{
Comparison of Direct Multiobjective Optimization Methods for the Design of Electric Vehicles
}

\author{
X. Roboam, J. Régnier, B. Sareni, Y. Ferfermann \\ LEEI, UMR INPT-ENSEEIHT/CNRS No. 5828 - 2 rue Camichel - BP 7122 - 31071 Toulouse Cedex 7 -France \\ e-mail: Xavier.Roboam@leei.enseeiht.fr
}

\begin{abstract}
"System design oriented methodologies" are discussed in this paper through the comparison of multiobjective optimization methods applied to heterogeneous devices in electrical engineering. Avoiding criteria function derivatives, direct optimization algorithms are used. In particular, deterministic geometric methods such as the Hooke \& Jeeves heuristic approach are compared with stochastic evolutionary algorithms (Pareto genetic algorithms). Different issues relative to convergence rapidity and robustness on mixed (continuous/discrete), constrained and multiobjective problems are discussed. A typical electrical engineering heterogeneous and multidisciplinary system is considered as a case study: the motor drive of an electric vehicle. Some results emphasize the capacity of each approach to facilitate system analysis and particularly to display couplings between optimization parameters, constraints, objectives and the driving mission.
\end{abstract}

Keywords : Heterogeneous systems, design, optimization, evolutionary algorithms, Hooke \& Jeeves, electric vehicle.

\section{Introduction}

Today, an overall viewpoint in the design of devices, a so called "system approach", is imposed by couplings between physical fields and the existence of multiple interactions between sub-systems. Indeed, concerning static and electromechanical converters or control systems, numerous design issues are more and more mastered when each element is separately designed. However, where the system design of heterogeneous devices is concerned, global optimization with respect to multiple objectives is useful and often necessary to achieve the best trade offs.

This paper then concerns the multiobjective optimization of electrical engineering systems by direct methods not related to the analytical calculation of the gradient. These techniques are applied to the drive part of a typical electric vehicle.

After a state of art classification of optimization methods is proposed, two completely different direct optimization methods are described in section III. To compare these approaches, we propose to apply them to a typical case study in electrical engineering: the embedded part of an electric vehicle. For that purpose, a system oriented modeling is presented whose characteristics (accuracy, validity domain) are directly linked to the optimization problem. The parameters (supply voltage, switching frequency, and geometrical motor parameters) can be fitted to all parts of the system with the view to obtaining a global optimum. The considered objectives are based on system losses, embedded mass and the cost of filtering devices. 
This case study is strongly non-linear. Thus, considering that the process can not be analytically derived, the objectives and the constraints have to be evaluated in a simulation procedure. The use of direct optimization methods then appears to be suitable to this problem.

In the last section, a deterministic geometric optimization algorithm (i.e. the Hooke and Jeeves algorithm [1]) is compared with a global and stochastic evolutionary algorithm (i.e. the second version of the Non-dominated Sorting Genetic Algorithm [2]). Some optimization results obtained with Hooke and Jeeves are presented. It enables underlining couplings between sub-systems which constitute a significant issue of the system analysis. They also show the interest of a global approach in the design of electrical engineering systems.

Finally, several performance issues are discussed and compared such as implementation costs (normalization and aggregation of objectives, constraints handling), the spread of optimal solutions in the objective space and the accuracy of results.

\section{Multiobjective Optimization Problems}

Multiobjective optimization seeks to simultaneously minimize $n$ objectives where each of them is a function of a vector $\mathrm{x}$ of $m$ parameters (or design variables). These parameters may also be subject to $k$ inequality constraints, so that the optimization problem may be expressed as :

$$
\begin{array}{ll}
\text { Minimize } & f(\mathbf{x})=\left(f_{1}(\mathbf{x}), f_{2}(\mathbf{x}), \cdots, f_{n}(\mathbf{x})\right) \\
\text { subject to } & g_{i}(\mathbf{x}) \leq 0 \text { for } i=1 . . k
\end{array}
$$

For this kind of problem, objectives typically conflict with each other. Thus, in most cases, it is impossible to obtain the global minimum at the same point for all objectives. Therefore, the problem has no single optimal solution but a set of efficient solutions representing the best trade-offs. These solutions consist of all design variable vectors for which the corresponding objective vectors cannot be improved in any dimension without disimprovement in another. They are known as Pareto-optimal solutions in reference to the famous economist. Mathematically, Pareto-optimality can be expressed in terms of Pareto dominance. Consider two vectors $\mathrm{x}$ and $\mathrm{y}$ from the design variable space. Then, $\mathbf{x}$ is said to dominate $\mathbf{y}$ if and only if [7] :

$$
\text { and } \quad \exists i=1 . . n \quad f_{i}(\mathbf{x}) \leq f_{i}(\mathbf{y})
$$

All design variable vectors which are not dominated by any other vector of a given set are called nondominated regarding this set. The design variable vectors that are non-dominated over the entire search space are Pareto-optimal solutions and constitute the Pareto-optimal front.

\section{Multiobjective optimization Methods}

Multiobjective optimization methods aim at finding one or multiple Pareto-optimal solutions to a particular optimization problem. Various multiobjective approaches can be used to guide the Decision Maker to a final solution among the Pareto-optimal set. A classification of these approaches is given below.

- A priori approaches (decide $\rightarrow$ search) for which the decision maker combines the different objectives into a scalar function then searches for only one Pareto optimal solution using singleobjective optimization methods. In particular, we will see how the geometric algorithms presented hereafter are based on an aggregation of all objectives. 
- Progressive approaches (decide $\leftrightarrow$ search) for which decision making and optimization are intertwined. The preferences of the decision maker are sequentially updated;

- A posteriori approaches (search $\rightarrow$ decide) for which the decision maker is presented with a set of efficient candidate solutions and chooses from that set. Pareto based Evolutionary Algorithms considered in this paper can be classified in this branch.

For optimization of heterogeneous systems, a fully analytical derivation is difficult, sometimes even impossible. Furthermore, electrical engineering systems include devices such as static or electromechanical converters whose noise level cannot be neglected. For both reasons, we have chosen to use direct search optimization methods to avoid numerical differentiation of objectives. In particular, we compare two opposite techniques in terms of decision making and optimization procedure (see Fig.1).

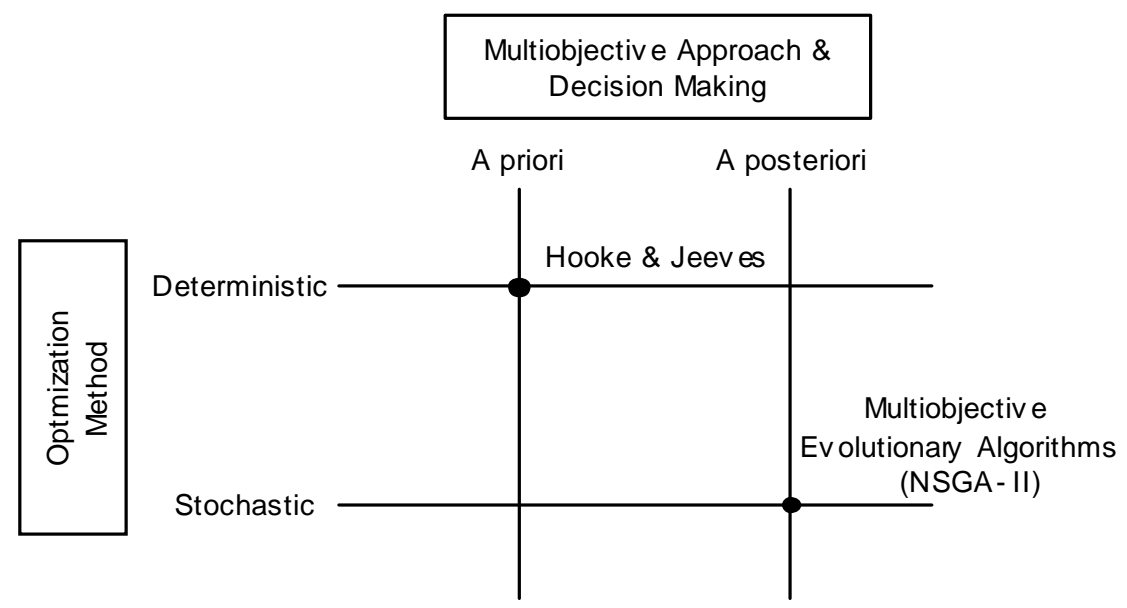

Fig.1: Investigated optimization methods

\section{III.1. Multiobjective optimization with deterministic geometric algorithms and objective aggregation}

The pattern search method of Hooke and Jeeves (HJ) [1] has been tested as geometrical method. Its principle involves two successive steps:

- The exploration step: From an initial parameter vector $\mathbf{x}_{0}$ (i.e. the reference point in parameter space), the algorithm processes an exploration search, displacing each parameter one by one with an increment $\left(+\Delta_{i}\right)$ while other parameters remain fixed. If the cost (i.e. objective) function is decreased (i.e. improved), then the new value of the parameter is preserved. On the contrary, if the objective function is disimproved, a negative increment $\left(-\Delta_{i}\right)$ is proposed to move this parameter; if both increments $\left(+\Delta_{i}\right.$ and $\left.-\Delta_{i}\right)$ do not offer any improvement of the objective function, each parameter remains unchanged. At the end of the process, each component of the parameter vector has been moved at least once and the cost function must be either decreased or unchanged. In this latter case, the process is reiterated with a smaller increment (typically $\Delta_{i} / 2$ ).

- The extrapolation step: when the exploration step is successful (i.e the objective function has been decreased by at least one positive or negative increment $\Delta_{i}$ ) a new point $\mathbf{x}_{e}$ is obtained and used with the last reference point to define the new reference point as follows:

$$
\mathbf{x}_{0} \leftarrow 2 \mathbf{x}_{e}-\mathbf{x}_{0}
$$

These steps are iterated until all increments $\Delta_{\mathrm{i}}$ have reached a given accuracy. 
In addition, we have to face a multiobjective problem [4]. It is advisable to scale the objectives so that they vary between 0 and 1 (see Fig. 2).

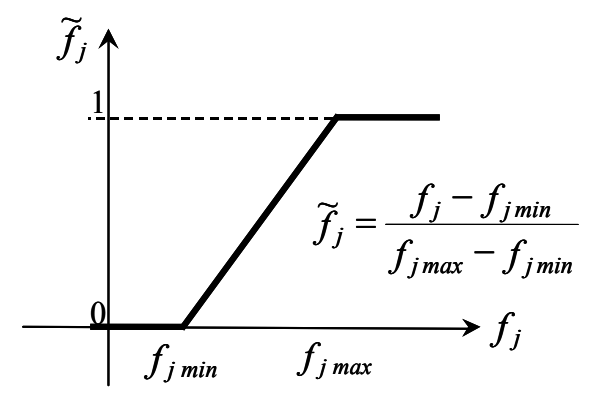

Fig.2: Objective scaling

Note that the same process applies for design variables and scaled parameters must be replaced by their physical values after the optimization step (i.e. physical process earlier represented in Fig 10). Moreover, searching minimum $f_{j \min }$ and maximum $f_{\text {jmax }}$ function values may require as many singleobjective optimizations as objectives. Aggregating each objective can be made by using weighting coefficients $\left(\alpha_{\mathrm{j}}\right)$ to achieve a global quality function.

$$
f=\sum_{j=1}^{n} \alpha_{j} \widetilde{f}_{j} \quad \text { with } \quad \sum_{j=1}^{n} \alpha_{j}=1
$$

where $n$ denotes the number of objectives. Generally, and more especially as electrical engineering heterogeneous systems are concerned, many constraints must be handled in non-linear programming problems. The Constrained Problem (CP) can be transformed into an Unconstrained Problem (UP) using the exterior penalty method (see Fig.3).

$$
\begin{gathered}
C P)\left\{\begin{array}{l}
\min (f(x)) \\
g(x) \leq 0 \\
x \in \mathfrak{R}^{\mathrm{n}}
\end{array}\right. \\
\longrightarrow(U P)\left\{\begin{array}{l}
\left.\min _{\varphi(x)}(x)\right) \\
\varphi(x)=f(x)+r . H(x) \\
x \in \mathfrak{R}^{\mathrm{n}}
\end{array}\right.
\end{gathered}
$$

Fig.3: Transformation of a constrained problem $(C P)$ into an unconstrained problem $(U P)$ using the exterior penalty method

A simple heuristic to calculate penalty coefficients $(r)$ has been put forward in [5] to increase the algorithm robustness. Using this procedure, a scalar objective function $\varphi(x)$ which integrates all constraints is then obtained and constitutes the input of the optimization procedure.

\section{III.2. Multiobjective optimization with Pareto Genetic Algorithms}

Contrary to the previous approach, Pareto Genetic Algorithms (GAs) lead to a formulation of the optimization problem without aggregating and normalizing objective functions. In the same way, integration of constraints is done independently of objective functions. GAs have been applied 
successfully to solve multiobjective optimization problems [6], [7]. Multiple solutions can be explored in parallel thanks to selection and evolution operators [8], [9]. Standard GAs can be modified to find the Pareto-optimal front of a multiobjective problem by using a specific selection method based on Pareto dominance rules (typically based on (2)). For constraint problems, these rules are generally extended to characterize the dominance of a candidate solution in relation to another solution of the search space. In our work, constraints are taken into account as follows:

- if the two candidate solutions are non-feasible, the Pareto-dominance is evaluated in the constraint space;

- if the two candidate solutions are feasible, the Pareto-dominance is evaluated in the objective space;

- if one candidate solution is feasible and the other not, the feasible solution dominates the nonfeasible solution.

From a randomly initialized population (i.e. set of individuals), a Pareto GA evaluates the nondominated solutions and preserves them in a specific archive (non-dominated set). For each generation, Pareto tournaments are used to select individuals from the archive to create the mating pool (parents of the current generation). Parents are crossed and mutated [8] to explore new solutions (children of the current generation). In this way, the population of children and the archive are merged to assess the non-dominated set of the next generation. If the number of non-dominated individuals is higher than the size of the archive, a clustering method is used to preserve most representative solutions and eliminate others in order to keep a constant archive size. Note also that niching is used in the selection scheme when individuals involved in a tournament have the same Pareto dominance ranking. The structure of a Pareto GA is depicted in Fig. 4.

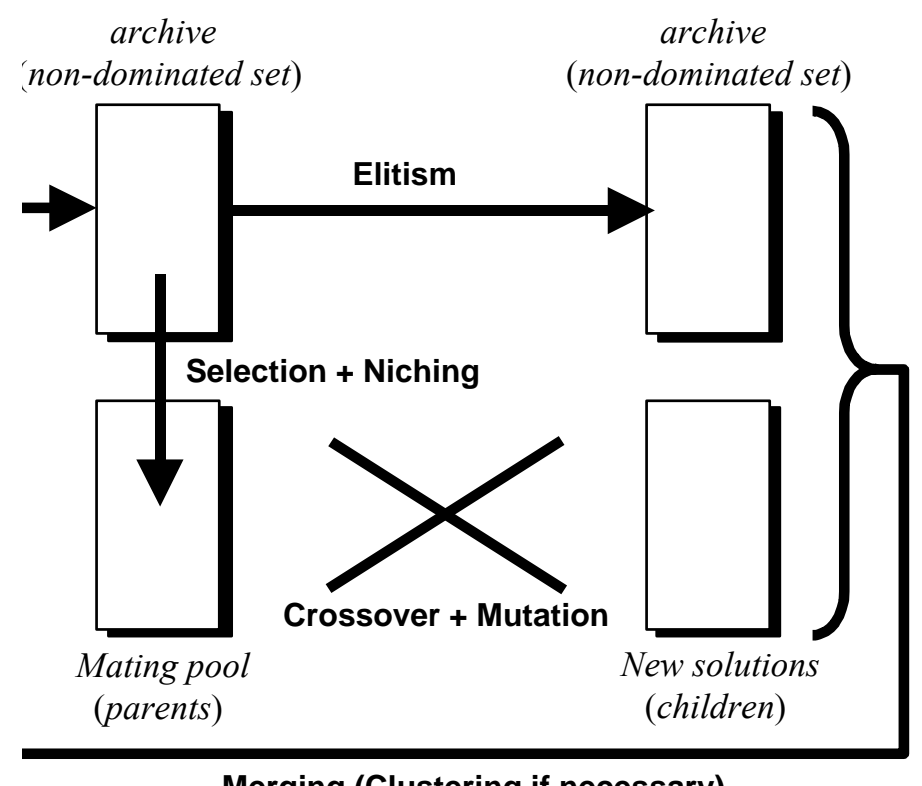

Merging (Clustering if necessary)

Fig.4: Structure of a Pareto GA (one step generation)

In this work, we have used a particular Pareto GA, which is the second version of the Non-dominated Sorting Genetic Algorithm (NSGA-II). This algorithm is based on the principles of Pareto GA's previously explained. In the NSGA-II, selection is performed with Pareto ranking tournaments associated with a crowded comparison operator to induce niching in the objective space. NSGA-II 
determines all successive fronts in the population (the best front corresponding to the non-dominated set). Moreover, a crowding distance is used to estimate the density of solutions surrounding each individual on a given front. In a tournament, if individuals belong to the same front, the selected individual is the one with the greater crowding distance. This niching index is also used in the clustering operator to uniformly distribute the individuals on the Pareto-optimal front. All details of this algorithm can be found in [2]. We have suggested some adaptations [10], more particularly devoted to increase the robustness from a self-adaptive recombination scheme used for crossover operators.

\section{System Modeling and Requirements}

A representative electromechanical device has been chosen as a case study in order to discuss the interest of optimization methods for the system design in electrical engineering. This section also allows emphasizing the complexity of establishing a whole model valid inside the overall parameter range and achieving a trade-off between low cost computation and accuracy respecting physical meaning: based on this trade-off "just enough accurate modeling" must be defined. For that purpose, we have selected the device represented in Fig.5, whose behavior is relatively well known and which allows understanding relations between system parameters, constraints, design objectives and the driving mission. Thus, we have considered an assembly including a battery supply, a low-pass input filter, a Voltage Source Inverter (VSI) feeding a PM brushless synchronous machine with trapezoidal electromotive forces (e.m.f) driving a mechanical load through a gearbox (speed reducer). This system is rated for a typical full electric vehicle application even if its modeling is simplified. This process is specified and optimized for a given driving mission (a urban mission has been chosen) that defines a speed reference and a loading torque (see Fig.6).

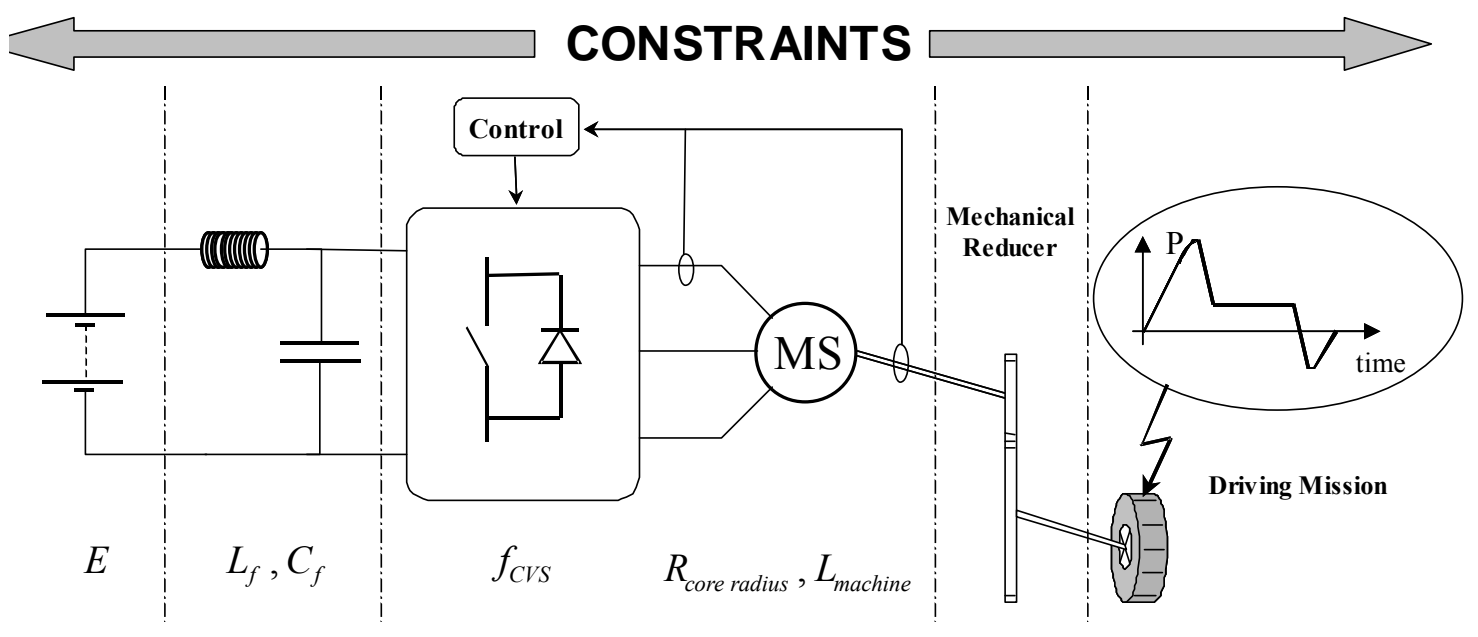

Fig.5: Synoptic of a the motor drive part of an electric vehicle 


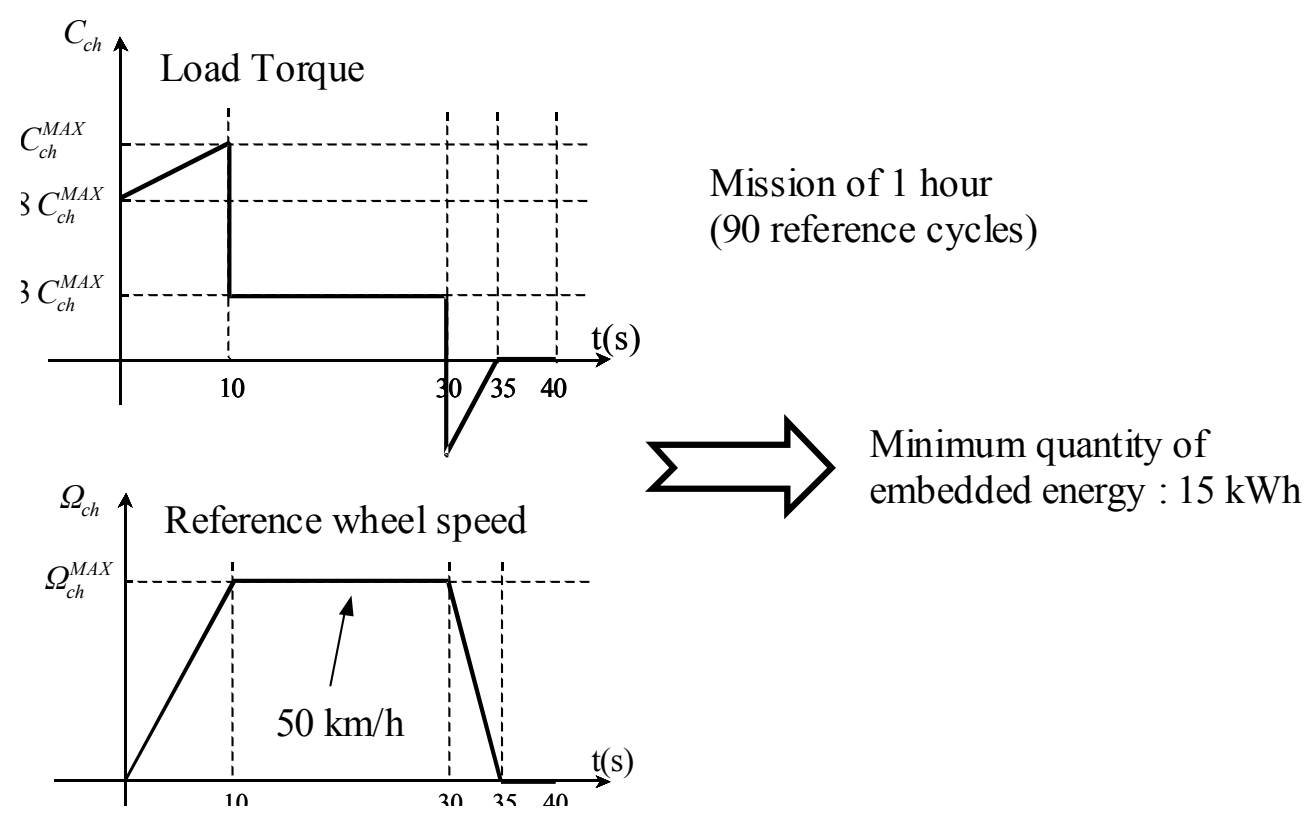

Fig.6: The urban driving mission

\section{IV.1. Definition of a urban driving mission}

An urban mission has been chosen to optimize this system. The vehicle speed is not greater than 50 $\mathrm{km} / \mathrm{h}$. A speed control loop which "simulates" the driver is included to impose the speed cycle represented in Fig. 6.

\section{IV.2. The Battery Model}

Being the most commonly used in electric vehicles, a pack of $160 \mathrm{Ah}$ lead acid battery is considered [18]. The model is simplified to be compatible with a global optimization process. One simplification hypothesis deals with the internal resistance which can be considered as constant if the discharge depth is limited to $75 \%$. Based on the vehicle requirements (see Fig.6) and on an average system efficiency, a preliminary analysis shows that $20 \mathrm{kWh}$ of energy are required for one hour of operation, which involves a minimum of 57 elementary cells. Given that an integer number of cells must be placed in a serial/parallel architecture, a variable DC voltage causes a non-linear variation of the cell number as illustrated in Fig 7.b.
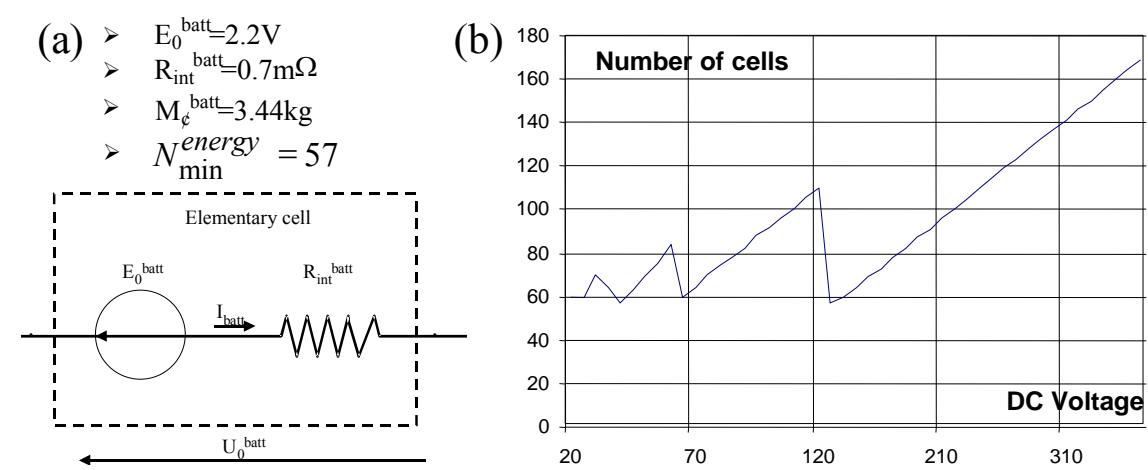

Fig.7: (a) Battery model and ratings (b) Number of cells versus DC voltage 


\section{IV.3. The Input Filter}

If the circuit model of the input filter is rather simple, its sizing is more complex when variable switching frequency, DC voltage and current have to be automatically handled during the convergence of the optimization procedure. Indeed, it is demanded that this element remains stable throughout the parameter range. But it is not a priori certainty since the torque controlled drive leads to drawing a constant power at a given speed, which can provoke instability of such systems. Furthermore, the rating of filter parameters is tuned on line with respect to constraints on the capacitor voltage and inductance current ripples. The oscillations constraints (6), (7) should be added with a frequency constraint (5): indeed, a sufficient ratio is demanded between the filter frequency and the switching frequency of the VSI $\left(f_{C V S}\right)$. For the considered modulation mode (see section IV.4), these constraints can be analytically derived:

$$
\begin{gathered}
f_{\text {filter }}=\frac{1}{2 \cdot \pi \cdot \sqrt{L C}}=\frac{f_{C V S}}{k} \\
\max _{\alpha=\frac{1}{2}}\left[\Delta U_{C}\right]=\frac{I_{c h}}{4 \cdot C \cdot f_{C V S}}
\end{gathered}
$$

where $I_{c h}$ denotes the motor current amplitude.

$$
\Delta I_{L}=\frac{\Delta U_{C}}{8 \cdot L \cdot f_{C V S}}
$$

A sufficient $k$ factor must be chosen to ensure the filtering quality. The choice of $L$ and $C$ values results from the waveform quality requirement.

Finally, a global cost of the input filter devices can be estimated regarding the cost / energy ratio:

$$
\text { filter cost : } \$=\alpha \cdot \frac{1}{2} \cdot L I^{2}+\beta \cdot \frac{1}{2} \cdot C E^{2}
$$

In fact, it is necessary to consider the input filter related criteria in order to establish a trade-off with the constraints of (5), (6), (7).

\section{IV.4. The VSI fed PMSM Motor with Trapezoidal EMF}

A Permanent Magnet Synchronous Motor (PMSM) with trapezoidal electromotive forces (emf) is considered. This kind of motor drive is widely used in traction applications due to its efficiency and its simplicity (low cost mechanical sensors...). In such a structure, $120^{\circ}$ rectangular currents must be imposed by the control unit. We have chosen a one transistor modulation which leads to minimizing switching losses and current ripple in the inverter. In order to simplify the model and to make it compatible with a system optimization procedure, space harmonic effects have been neglected in the machine. This latter hypothesis is acceptable with magnets whose shape perfectly creates trapezoidal emf. In the same way, current switching times $\left(120^{\circ}\right.$ currents) are neglected, so that the PMSM behavior is similar to that of a "simple DC motor". In fact, these simplifications are fulfilled with respect to the considered optimization criteria (losses, mass...) which are described below.

An average model of the PWM based on the duty cycle $(\alpha)$ is chosen to limit the computation time. A classical cascaded speed - current control is implemented to simulate the driving decisions satisfying the mission. Switching and conducting losses are estimated with reference to data sheets corresponding with the power switches (IGBT). Note that the switch class could be changed relatively to the DC voltage/current evolution during the optimization procedure. 

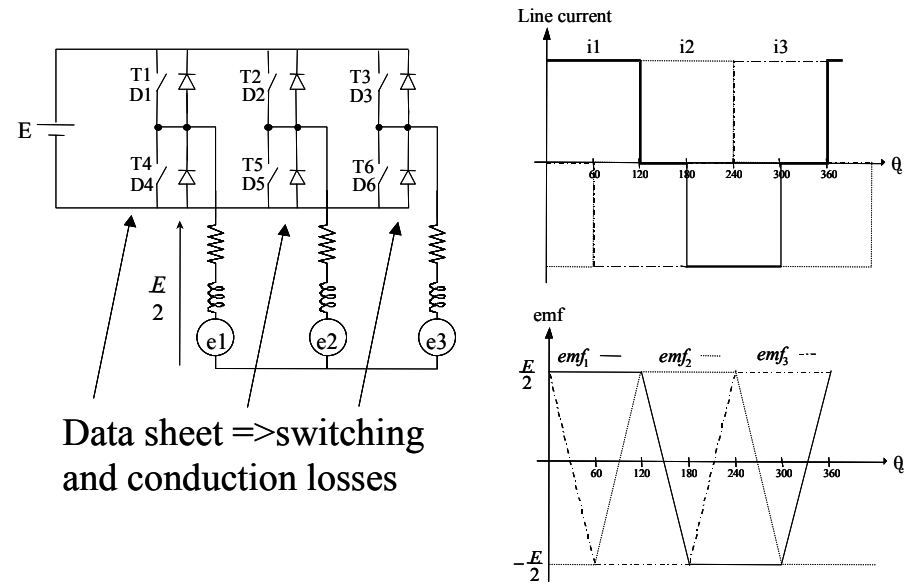

Fig.8: VSI fed PMSM with trapezoidal emf

\section{IV.5. The Permanent Magnet Brushless DC Motor Model}

From a given driving mission, changing some of the optimization parameters, as in particular the reducer ratio, also implies modifying the machine ratings by adapting the torque-speed characteristic. Including field computation from a Finite Element Analysis (FEA) in the optimization procedure is too expensive in computation time. It has led us to define a "Model of Synthesis based on Similarity" laws (MSS) which uses analytical equations and provides the advantage of being able to re-size a machine without using the F.E.A. The design process follows two steps:

- First, a "classical" analytical design or so called "Reference design" of the machine is made, fulfilling the torque-speed characteristic for the load requirements (reducer ratio and driving mission being given). Then, the FEA is used once to check these specifications.

- Secondly, from the "Reference machine", the MSS allows designing a new machine having the same structure, but including new geometrical dimensions (i.e. core radius $r_{\mathrm{s}}$ and length $l_{r}$ ). The aim of the MSS is to give dimensions and characteristics of a new machine which fulfills the required specifications such as torque-speed characteristic for the load.

The equations of the MSS are given in (9)

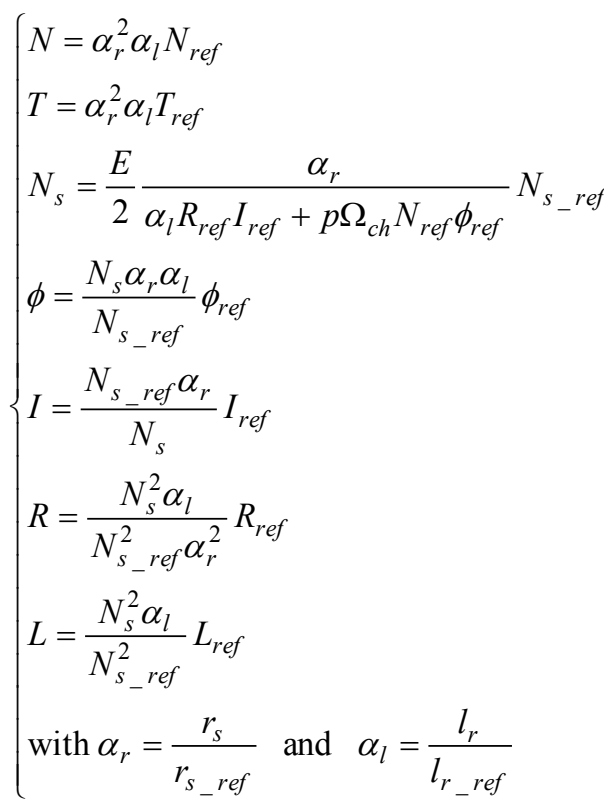


where the "ref" index applies for parameters of the reference design and with

$N$ : reducer ratio

$T:$ machine torque

$N_{s}$ : number of conductors

$E:$ DC voltage

$I / \phi:$ stator current/flux

$R / L:$ stator resistance/inductance

$r_{s} / l_{s}:$ core radius/machine length

With respect to the "reference machine", per unit geometrical parameters $\alpha_{r}$ and $\alpha_{l}$ are defined in (9). The global similarity relations take into account the variations of the conductor number and geometrical dimensions, and obviously enable fulfilling the chosen operating point in the torque-speed plane. Fig. 9 gives an overview of the MSS structure. A detailed description of this model is given in [12]. Its validation process from FEA is also proved.

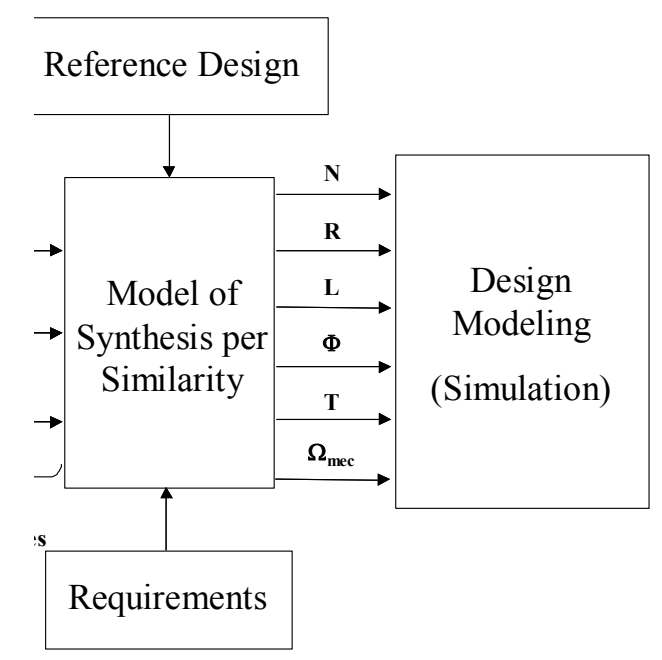

Fig.9: MSS Structure

An estimation of motor losses (copper, iron, and mechanical losses) is added to this model. It is based on analytical expressions. For the copper losses, the expression is rather classical:

$$
P_{\text {copper }}^{\text {Mach }}(t)=2 \cdot R \cdot I(t)^{2}
$$

where $R$ and $I$ are respectively the motor resistance and current. On the contrary, estimating iron losses (hysteresis and eddy currents) is very complex and research is still being carried out on this issue [13]. For the optimization process, hypothesis are made to simplify the analytical derivation: i.e. only stator losses are considered, the first harmonic approximation is made, hysteresis and Eddy current losses are globally estimated and a mathematical interpolation gives the following analytical equation :

$$
P_{\text {iron }}=0.0025 \cdot f^{1.45} \cdot B_{\max }^{1.91}
$$

Finally, a thermal constraint is verified for each criteria evaluation, by means of an equivalent thermal model of the machine. This model takes into account copper and iron losses as inputs. 


\section{System Optimization}

\section{V.1. Optimization Framework}

Based on the "system oriented model" previously described, different criteria allow qualifying the system's performance.

- global mass : battery + motor + vehicle (this latter part being fixed at $650 \mathrm{~kg}$ );

- total losses (copper and iron losses, conducting and switching losses, mechanical losses);

- estimated cost of the input filter;

The six system optimization parameters are:

- the DC supply voltage $(E)$,

- the capacitor $C_{f}$ and the inductor $L_{f}$ (input filter's devices),

- the switching frequency of the semiconductors $\left(F_{C V S}\right)$,

- the stator core radius $r_{s}$ and the length $l_{s}$ of the motor,

and their limit in the optimization process are given in Table 1.

Table 1: Optimization parameter range

\begin{tabular}{cl}
\hline Design Variables & \multicolumn{1}{c}{ Range } \\
\hline Battery Voltage & $E \in[20,500][\mathrm{V}]$ \\
\hline Filter Inductance & $L_{f} \in\left[10^{-5}, 0.005\right][\mathrm{H}]$ \\
\hline Filter Capacitor & $C_{f} \in\left[10^{-4}, 0.003\right][\mathrm{F}]$ \\
\hline Converter Switching Frequency & $F_{C V S} \in[500,10000][\mathrm{Hz}]$ \\
\hline Motor Core Radius & $r_{s} \in[0.05,0.2][\mathrm{m}]$ \\
\hline Motor Length & $l_{s} \in[0.05,0.4][\mathrm{m}]$ \\
\hline
\end{tabular}

Many constraints are also added to complete the optimization framework:

- constraints on parameter domain;

- constraints linking parameters between each other : for example $C_{f}, L_{f}, F_{C V S}$ are linked by constraints (see (5), (7)) relative to stability criteria and voltage/current ripples ;

- constraints resulting from the simulation : for example thermal constraint that verifies if a given machine can satisfy the driving mission from a thermal point of view (i.e. the temperature on copper windings should be lower than $150^{\circ}$ ).

This process of constraints handling is summarized in Fig. 10 in the case of the Hooke and Jeeves algorithm. The optimization process has almost a similar structure for evolutionary algorithm, except for normalization, constraint penalties and objective weightings. 


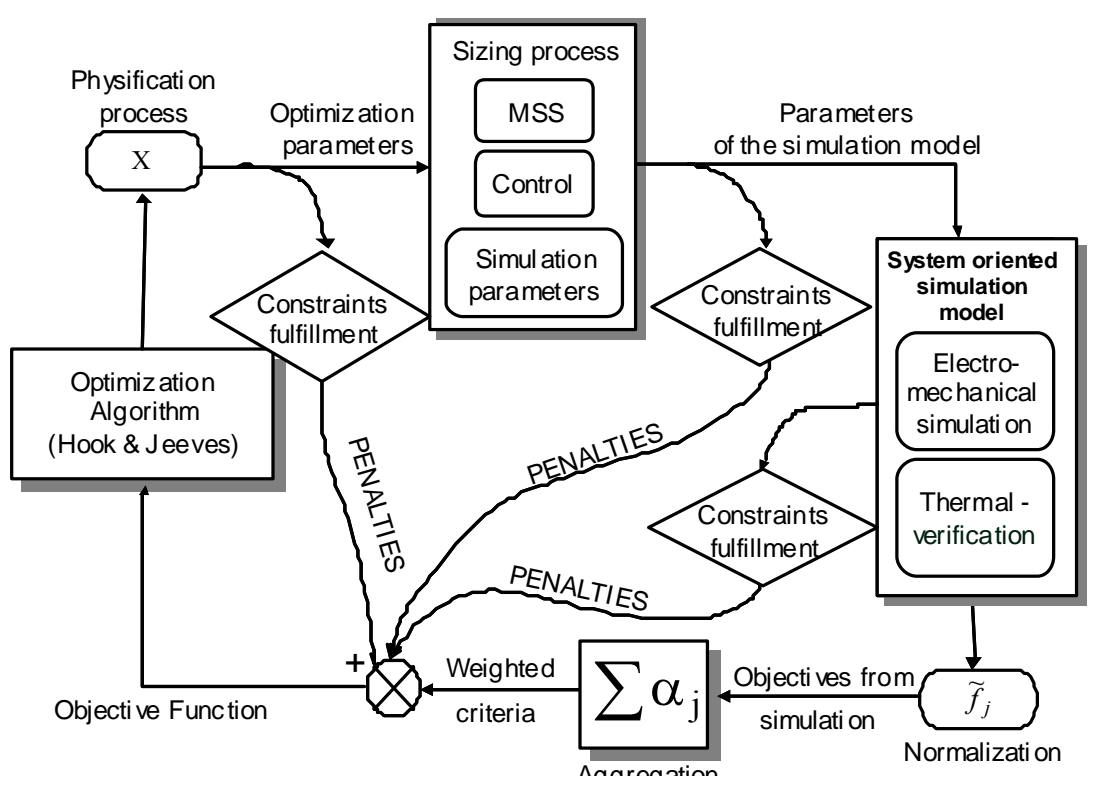

Fig.10: Synoptic of the optimization process (HJ method)

\section{V.2. Electric Vehicle Optimization with HJ}

Before comparing geometrical and evolutionary algorithms in the context of direct and multiobjective system optimization, let us begin with the HJ method and see how it has been practically applied to this system and its requirements [5]. The system analysis is certainly the most complex task during the optimization process, due to the important number of couplings between parameters - constraints - objectives - mission. In addition, representing results graphically is more and more complex as the criteria number increases. In order to facilitate this analysis, a 2-dimensional representation has been considered by limiting the optimization to only 3 weighting factors for the input filter cost : i.e. $\alpha_{\text {filter }}=0,0.33$ and 0.66 . Thus, the following results can be represented in the objective plane with respect to losses and mass criteria. The evolution of global mass and losses is obtained from several optimizations of the aggregated cost function with different balances of the two objectives: a sort of Paretooptimal solutions is then obtained. Fig. 11 superposes the three different Pareto-optimal fronts corresponding to the three different weighting factors of the input filter. It can be seen that this latter does not lead to significant changes of the Pareto-optimal front shape. 


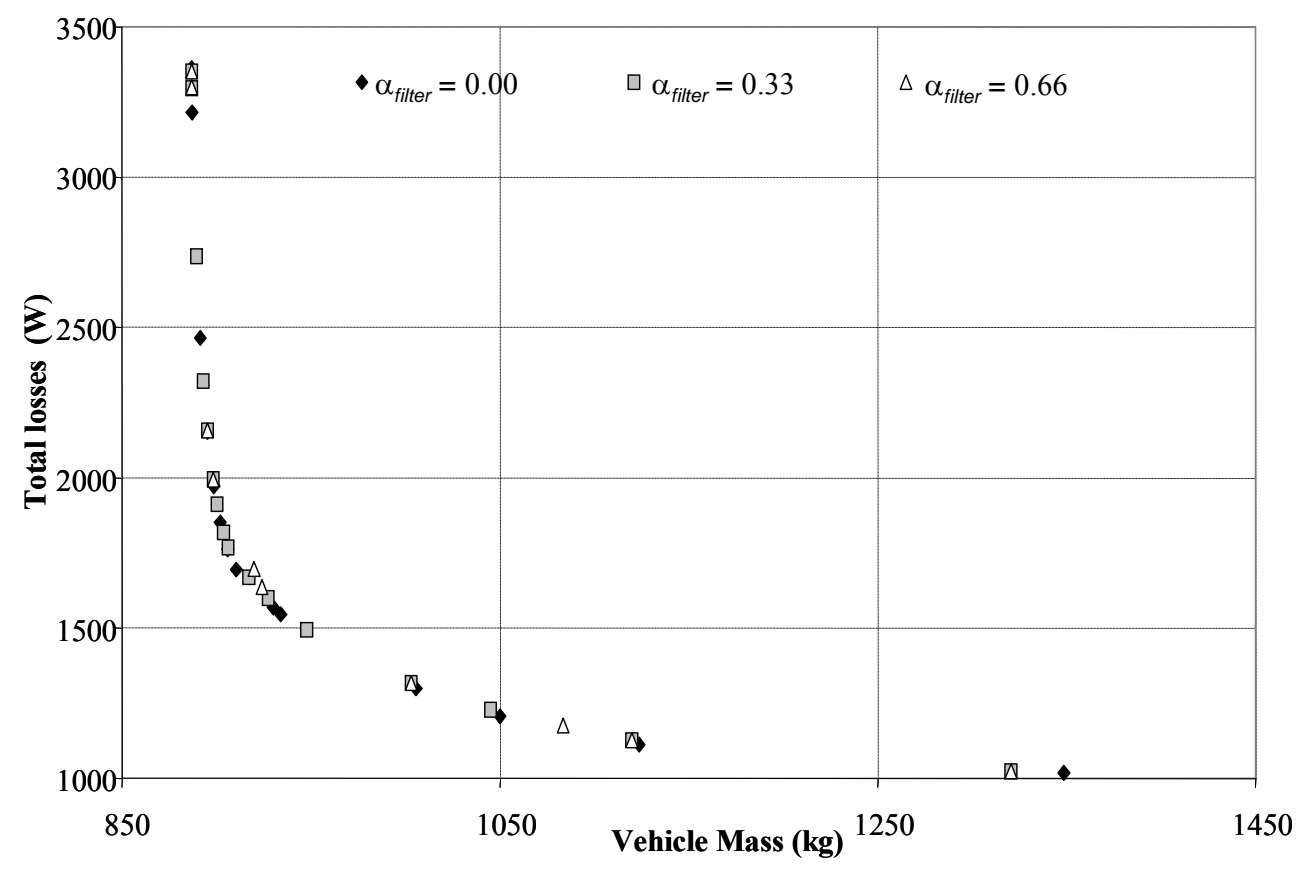

Fig.11: Pareto-optimal front for given weighting of input filter criterion

Fig.11 shows also that objective variations are significant:

- variation of losses from 1000 to $3400 \mathrm{~W}$

- global mass from $900 \mathrm{~kg}$ till $1350 \mathrm{~kg}$ (embedded mass from 250 to $700 \mathrm{~kg}$ ).

Note that the optimization problem statement is convenient and leads to significant trade-offs. We can observe that this latter issue is not so easy to obtain, as complex heterogeneous systems are considered in relation to several objectives. Each optimization of the aggregated criteria function (one point of the Pareto front) needs 1295 objective function evaluations on average with the HJ method. Considering that 14 different weighting coefficients are necessary to accurately define the Pareto front, more than 18000 objective function evaluations of the one-hour mission are necessary. In fact, this multiobjective optimization process is equivalent (in terms of computation cost) to the analysis of the electric vehicle system simulated for more than 2 years! At this level, a rigorous analysis of all provided information must be carried out:

- first, the global objective analysis is not sufficient and relative variations of "partial criteria" have to be considered. For the whole loss objective, the partial criteria are battery losses, the switching and conducting losses in the VSI, iron and copper losses, and finally mechanical losses (see Fig.12). In fact, examining amplitudes as well as the directions of these variations is very informative. For example, the shape of motor copper losses reveals a local optimum in the medium part of the Pareto-optimal front, so that it increases in the right part of the front while all other losses decrease in the same region like the global losses. More generally, this example emphasizes that the global optimum is rarely equivalent to the sum of local optima. 

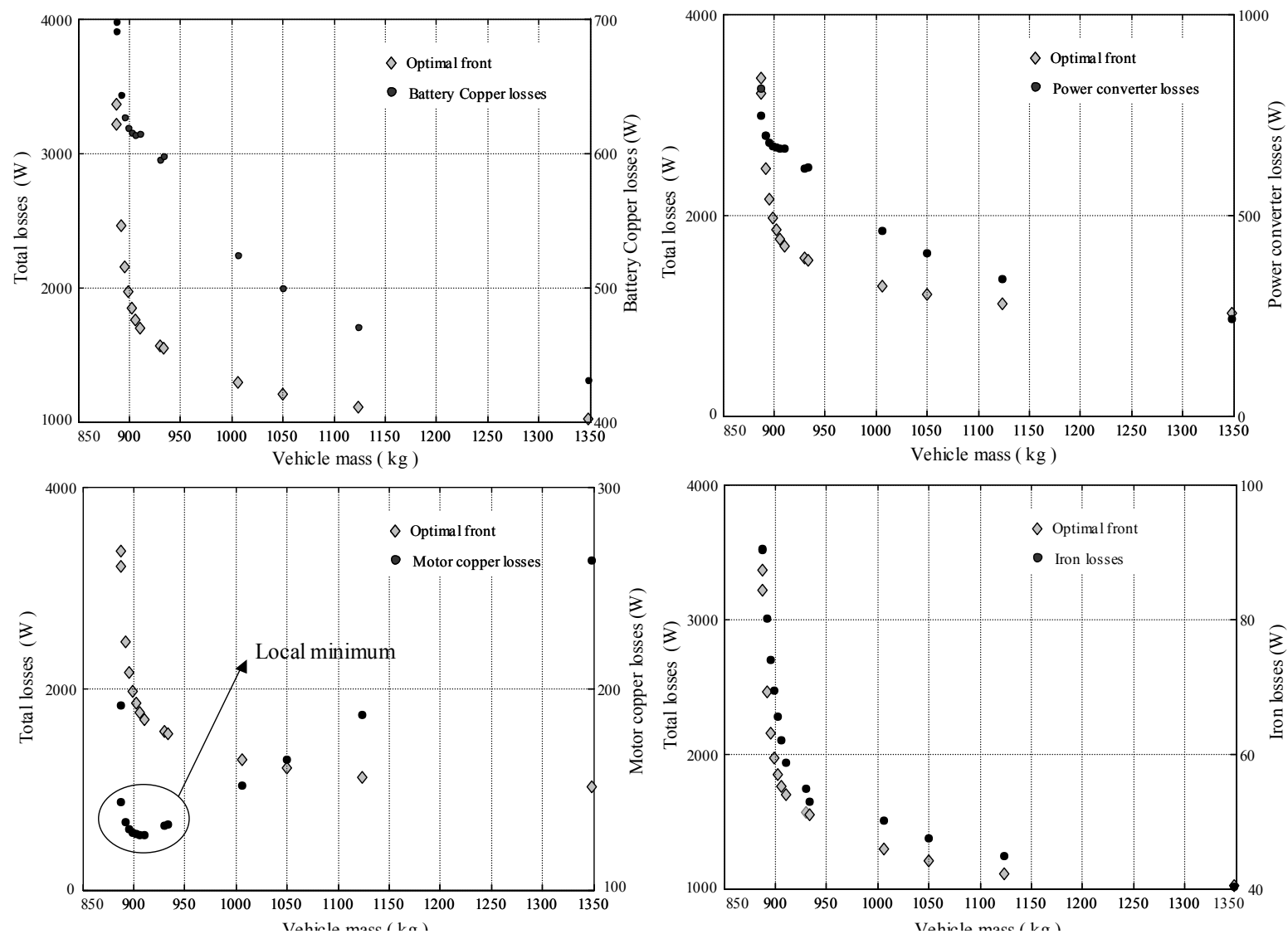

Fig.12: Partial criteria of Pareto-optimal solutions

- Secondly, the parameter evolution should be analyzed. Fig. 13 illustrates two of the optimization parameters for a null weighting factor of the input filter. Only the machine core radius and the input voltage have a significant influence. In fact, the others (i.e. the machine length and the switching frequency) are fixed by the optimizer to their minimum value. Machines with large radius over length ratio are naturally preferred to optimize the loss-mass trade-off. Let us note that wheel motors recently designed in traction systems have the same kind of geometry. On these particular tests, it can also be observed that the battery voltage rises in a linear way when the global mass is increased (battery mass increase) while losses are reduced. This latter fact can be explained by the reduction of the current level, which decreases converter and machine losses. Some parameters are close to constraints: the switching frequency is in this case decreased to minimize switching losses and is only limited by the frequency constraint (see (5)). 

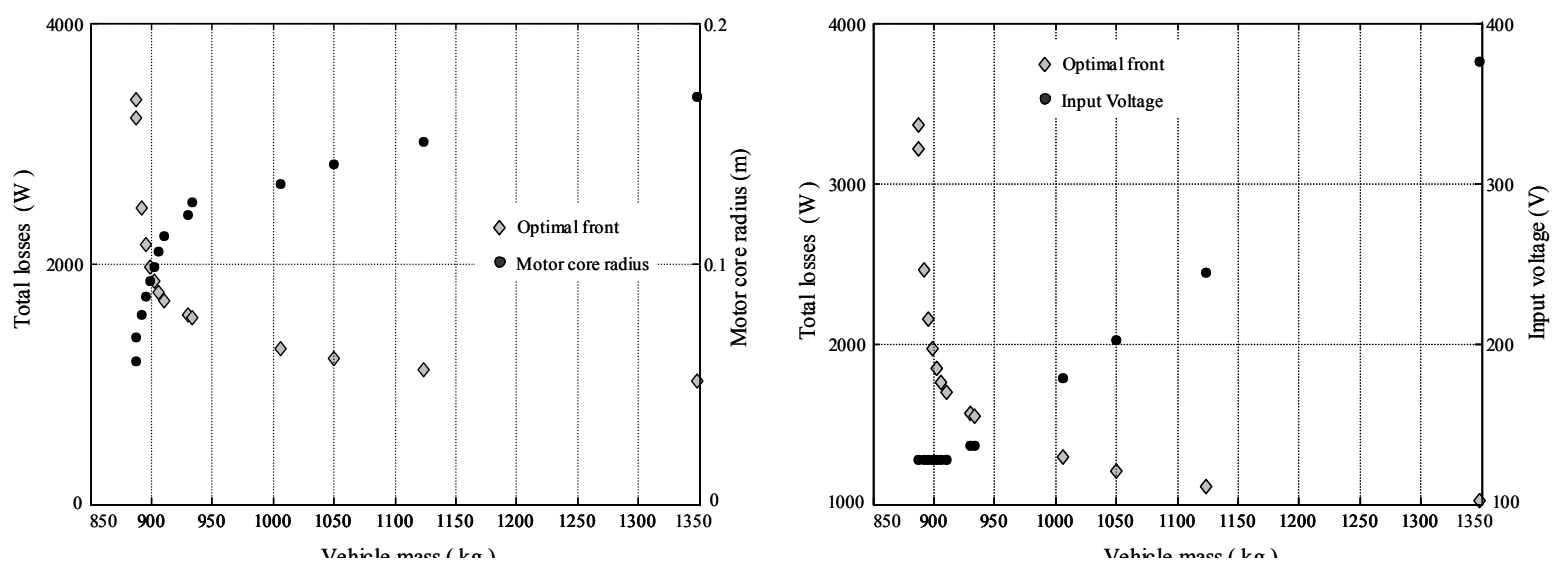

Fig.13: Motor core radius and input voltage evolution of Pareto-optimal solutions

The analysis of such results is really interesting for designers because it directly reveals system couplings between parameters - objectives - constraints and the driving mission. For example, we have observed that motor copper losses and embedded mass are coupled with each other through the driving mission and the vehicle dynamic. Indeed, following the frequency spectrum of the driving mission, frequent and high variations of the vehicle speed are provoked. The acceleration force $(M . d V / d t)$ is then all the more important that the embedded mass $M$ is high. Finally, resulting torques (i.e. current levels) lead to copper losses coupled with the mass which is embedded during the mission. This latter example emphasizes the interest of the so called "systemic design" which consists in an overall approach of the design including the system finality (mission).

\section{V.3. Geometric versus Evolutionary Optimization Algorithms}

In this section, both very different optimization methods are compared:

- for geometrical algorithms, the deterministic direct search pattern algorithm of Hooke \& Jeeves is considered as presented in section II.A.

- for multiobjective evolutionary algorithms, the NSGA-II with self-adaptive recombination used for crossover operators has been implemented as explained in section II.B.

The main differences between both approaches have been described in section II, particularly for differences between aggregative (a priori) and Pareto (a posteriori) search methods. Only comparing both algorithms in terms of convergence would be insufficient as a conclusion. The present objective is also to compare the results quality as well as complexity of algorithms implementation. Their radically different natures (deterministic versus stochastic) also have to be discussed.

\section{1) Implementation}

- HJ Method:

Conditioning of the optimization problem needs:

- tuning weighting coefficients;

- determining normalization factors (objectives and constraints); 
- tuning penalty coefficients;

The determination of normalization factors for objectives can be problematic as variation domains of objectives are a priori unknown or inaccurately predictable. This process generally needs preliminary single-objective optimizations to determine minimum and maximum values of each objective function. For issues related to constraints, different handling methods exist (exterior/interior penalties). Whatever the approach, choosing both constraint function $H(\mathbf{x})$ and penalty coefficient $r$ (see Fig.3), is often complex and sometimes affects the algorithm robustness. It also depends on the optimization problem characteristics. Furthermore, penalty coefficient and function could be different if constraints are relative to variation domain on design variables or to constraints involving several parameters of the objective function. Considering that the variation of constraints is difficult to predict in certain cases, constraint normalization also constitutes an obstacle in the algorithmic implementation. The spread of the solutions in the objective space must also be analyzed. Indeed, this latter issue depends on the distribution of weighting coefficient when aggregative methods are implemented. In fact, a linear variation of weighting coefficients does not guarantee a uniform distribution of solutions in the objective space.

- Multiobjective evolutionary algorithm NSGA-II:

In this case, optimization problem conditioning appears simpler than previously: neither normalization nor weighting coefficients are required thanks to the Pareto-dominance concept. In the same way, no penalty function or coefficient is necessary. Indeed, if a given constraint is not fulfilled, specific Pareto-dominance rules are applied (see section II.B). Contrary to aggregation based methods, the solutions are naturally spread along the Paretooptimal front thanks to the niching procedure. Furthermore, variation domains of optimization parameters are directly included in the algorithm: if a design variable goes beyond its validity range during a genetic operation (crossover or mutation), this variable is automatically adjusted according to the corresponding boundary.

\section{2) Comparative results}

The stochastic nature of the evolutionary algorithm requires performing the same optimization problem several times to assess the reproducibility of the results. For one optimization run, the number of objective function evaluations must be shared among the individual number $\left(N_{\text {ind }}\right)$ and the generation number $\left(N_{\text {gen }}\right)$. Consequently, the total number of objective function evaluations for $N_{\text {test }}$ independent runs is $N_{\text {test }} \times N_{\text {ind }} \times N_{\text {gen }}$. Thus, the tuning parameters of Table 2 have been selected for the NSGA-II leading to 17500 objective function evaluations for a fair comparison with the HJ procedure (remind that 14 optimization runs have been carried out with the HJ method leading to 18000 objective function evaluations). 
Table 2: NSGA-II tuning parameters

\begin{tabular}{ll}
\hline Number of independent runs $\left(N_{\text {test }}\right)$ & 5 \\
\hline Population size $\left(N_{\text {ind }}\right)$ & 50 \\
\hline Generation number $\left(N_{\text {gen }}\right)$ & 70 \\
\hline Mutation rate for $m$ design variables & $1 / m$ \\
\hline Mutation rate for the crossover $X$-gene $($ see $[10])$ & $5 \%$ \\
\hline
\end{tabular}

Fig. 14 shows that both methods converge to the same Pareto-optimal front. Note that the NSGA-II Pareto-front plotted in this figure corresponds to a concatenation of the five Paretofronts obtained from the five independent runs. However, these runs have provided similar results. Therefore, NSGA-II requires $50 \times 70=3500$ objective function evaluations to obtain fifteen Pareto-optimal solutions on this design problem. With the same number of objective function evaluations, the HJ procedure only offers two Pareto-optimal solutions. This confirms the greater exploration capability of the NSGA-II in comparison with local optimization methods.

The efficiency in the determination of the boundary solutions was almost equivalent with both investigated methods since the minimum loss solution has been found by the NSGA-II while the HJ procedure has detected the minimum mass solution. The performance of both optimization methods was also characterized by two quantitative criteria. The $\Delta$ spacing factor used in [2], [10] has been evaluated in each case to assess the quality in terms of distribution homogeneity along the Pareto-optimal front. This criterion is based on consecutive distances among the solutions of the Pareto-optimal front. It characterizes the capability of the optimization method to distribute its solutions uniformly along the Paretooptimal front. A value of zero for this metric indicates all non-dominated solutions found are equally spaced. Concerning the solution accuracy, the coverage index used in [6] was considered to compare NSGA-II and HJ efficiency.

The comparative results for both performance criteria are summarized in table 3. They show that NSGA-II ensures a better spread of Pareto-optimal solutions along the Pareto-optimal front thanks to the efficiency of its clustering operator. On the contrary, HJ procedure is characterized by a bad spread as a reason of a non-linear relation between weighting coefficients and Pareto-optimal solution distribution. Low coverage indexes indicate that NSGA-II and HJ fronts are non-covered. Most solutions found by the two different approaches are non-dominated. Consequently, the accuracy in the determination of Paretooptimal solutions was almost similar. 


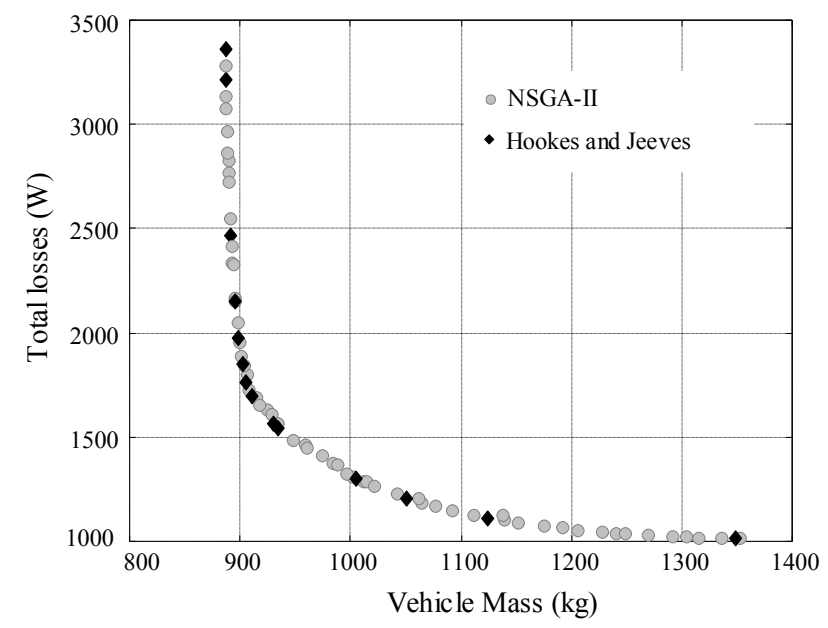

Fig.14: Pareto-optimal solution distribution in the objective space with HJ and NSGA-II

Table 3: Comparative results (spread and accuracy)

\begin{tabular}{ccc}
\hline Performance criterion & NSGA-II & HJ \\
\hline Spacing $\Delta$ & 0.744 & 1.219 \\
\hline Coverage index $($ see $[6])$ & $C($ NSGA-II/HJ $)=0.07$ & $C($ HJ/NSGA-II $)=0.08$ \\
\hline
\end{tabular}

\section{Conclusion}

In this paper, we have presented very different optimization techniques for constrained and multiobjective problems. These two families of methods have been applied to a typical (heterogeneous, non linear, including mixed variables) electrical engineering system: an embedded electrical actuator. For that purpose, a system-oriented whole model of the vehicle has been put forward. This model respects the trade-off between validity domain and constraints which have to be faced where a system optimization is concerned. Indeed, the optimization process has required a high number of objective function evaluations which correspond to more than two years of simulated time! The results given by each direct optimization method (i.e. the Hooke and Jeeves and evolutionary algorithms) show strong properties of convergence and robustness. The performance of each approach has been analyzed in terms of convergence, accuracy and diversity. With the same number of objective function evaluations, results have shown the NSGA-II superiority concerning the exploration capability and its tendency to find well spread Pareto-optimal solutions. Moreover, the NSGA-II accuracy was comparable with the well-known efficiency of the HJ deterministic procedure.

The adaptation of this approach on more complicated systems (e.g. hybrid vehicles) essentially concerns modeling aspects. Optimization algorithms such as genetic algorithms can be easily applied on this kind of systems with combinatorial features. In this case, we recommend adding, in the optimization process, discrete parameters related to architecture variations and energy management strategies to sizing design variables.

\section{References}

[1] R. Hooke and T. Jeeves, "Direct Search Solution of Numerical and Statistical Problems," Journal of the ACM, Vol. 8, p. 212-229, 1961. 
[2] K. Deb, S. Agrawal, A. Pratab, T. Meyarivan, "A fast-elitist non-dominated sorting genetic algorithm for multiobjective optimization: NSGA-II," Proceeding of the Parallel Problem Solving from Nature VI Conference, pp. 849-858, 2000.

[3] J. A. Nelder and R. Mead, "A simplex Method for Function Minimization," Computer Journal, Vol. 7, p. 308-313, 1965.

[4] A. Törn, A. Zilinskas, "Global optimization," Lecture Notes in Computer Sciences, Vol. 350, Springer Verlag, 1989.

[5] Y. Fefermann, Optimisation directe de systèmes hétérogènes en Génie Electrique, PHD thesis of INP Toulouse, France, December 2002.

[6] E. Zitzler, Evolutionary Algorithms for Multiobjective Optimization: Methods and Applications, $\mathrm{PhD}$ thesis, Swiss Federal Institute of Technology (ETH), Zurich, Switzerland, 1999.

[7] J. Horn, N. Nafpliotis, D. Golberg, "A niched Pareto Genetic Algorithm for multiobjective optimization," First IEEE Conference on Evolutionary Computation, IEEE World Congress on Computational Intelligence, Vol. 1, pp 82-87, 1994.

[8] D. Golberg, Genetic Algorithms in Search, Optimization and Machine Learning, Reading, MA: Addison-Wesley, 1989.

[10] B. Sareni, J. Régnier, X. Roboam, "Recombination and Self-Adaptation in Multi-Objective Genetic Algorithms,",Lectures Note in Computer Science, Vol. 2936, P. Liardet et al Ed, pp.115-126, Springer Verlag, 2004.

[12] Y. Fefermann, S. A. Randi, S. Astier, X. Roboam, "Synthesis models of PM Brushless Motors for the design of complex and heterogeneous system," EPE'01, Graz, Austria, September 2001.

[13] K. Attalah, Z.Q. Zhu, D. Howe, "The prediction of iron losses in Bruhless permanent magnet DC motors," International Conference on Electrical Machines, ICEM'92, Manchester, England, 1992.

[14] J. Régnier , B. Sareni, X. Roboam, S. Astier, "Optimal design of electrical engineering systems using Pareto Genetic Algorithms," EPE’03, September 2003, Toulouse, France.

[15] Y. Fefermann, X. Roboam, J.M. Enjalbert, "Direct optimization of Motor Drives systems for an electric vehicle," IEEE ISIE’04, Ajaccio, France, May 2004.

[16] S. A. Randi, S. Astier, B. Sareni, "Full modelling approach of electric vehicle for design optimization," EVS 18th conference, Berlin, Germany, Oct 21-24, 2001.

[17] J. S. Chun, J.P. Lim, J.S. Yoon, "Optimal design of synchronous motor with parameter correction using immune algorithm," IEEE Trans on Energy Conversion, Vol 14 №3 pp 610-615, September 1999.

[18] L.M. Manninen, R.H. Hutka, P.D. Lund, "Wind diesel system analysis with special emphasis on battery modelling", EWEC'91, Elsevier Science Publishers, 1991. 